\title{
Magnetic Resonance Imaging Simulator: A Teaching Tool for Radiology
}

\author{
Debra Rundle, Sheel Kishore, Sridhar Seshadri, and Felix Wehrli
}

\begin{abstract}
The increasing use of magnetic resonance imaging (MRI) as a clinical modality has put an enormous burden on medical institutions to cost effectively teach MRI scanning techniques to technologists and physicians. Since MRI scanner time is a scarce resource, it would be ideal if the teaching could be effectively performed off-line. In order to meet this goal, the radiology Department at the University of Pennsylvania has designed and developed a Magnetic Resonance imaging Simulator. The simulator in its current implementation mimics the General Electric Signa (General Electric Magnetic Resonance Imaging System, Milwaukee, WI) scanner's user interface for image acquisition. The design is general enough to be applied to other MAI scanners. One unique feature of the simulator is its incorporation of an image-synthesis module that permits the user to derive images for any arbitrary combination of pulsing parameters for spin-echo, gradient-echo, and inversion recovery pulse sequences. These images are computed in 5 seconds. The development platform chosen is a standard Apple Macintosh II (Apple Computer, Inc, Cupertino, CA) computer with no specialized hardware peripherals. The user interface is implemented in HyperCard (Apple Computer Inc. Cupertino, CA 1. All other software development including synthesis and display functions are implemented under the Macintosh Programmer's Workshop ' $C$ ' environment. The scan parameters, demographics, and images are tracked using an Oracie (Oracle Corp, Redwood Shores, CA ) data base. Images are currently stored on magnetic disk but could be stored on optical media with minimal effort. (C) 1990 by W.B. Saunders Company.
\end{abstract}

KEY WORDS: magnetic resonance imaging, teaching. computers, images, simulator, database, synthesis.

From the Department of Radiology, Hospital of the University of Pennsylvania, Philadelphia.

Supported in part by a technology grant from the Ben Franklin Partnership of the Advanced Technology Center of Southeastern PA, Grant No. 07.944.

This report appeared previously in Society of Photooptical Instrumentation Engineers proceedings on Medical Imaging $I V$.

Address reprint requests to Ms Debra Rundle. Hospital of the University of Pennsylvania, Department of Radiology, 3400 Spruce St, Philadelphia, PA 19104.

0 1990 by Society of Photo-optical Instrumentation Engineers.

Signa-Tutor is available through the Center for Continuing Education, Hospital of the University of Pennsylvania, Department of Radiology, 3440 Market St, Philadelphia, PA 19104

0897-1889/90/0304-0017\$03.00/0
$\mathbf{I}^{\mathrm{N}}$ N THE LAST SEVERAL years, magnetic resonance imaging (MRI) has gained a clinical stronghold in radiological imaging. With an installation cost per clinical imaging system exceeding two million dollars, it is imperative that the system be used to its fullest extent. As a result, accessibility to the scanner for teaching purposes is minimal. To alleviate these constraining time and cost factors, an MRI simulator called Signa Tutor was developed at the Hospital of the University of Pennsylvania. Signa Tutor is an off-line simulation of the General Electric Signa (General Electric Magnetic Resonance Imaging System, Milwaukee, WI) data acquisition console. It is intended for teaching MRI acquisition concepts to technicians, clinicians, and residents away from the clinical site.

\section{DESIGN}

The Signa-Tutor is a HyperCard (Apple Computer, Inc, Cupertino, CA) application stack written for the Apple Macintosh (Apple Computer, Inc, Cupertino, CA) computer. Currently, it runs on any Macintosh II family or SE 30 model computer, provided it is equipped with 8 bit extended video (permitting 256 levels of gray). The storage requirement is at least four Mbytes of RAM and a 40 Mbyte hard drive. No other special equipment is required. However, if image transfer is desired, a network link can be established using Ethernet (Novell, Inc, Walnut Creek, CA) on both a Macintosh II and the Signa's host computer (Data General MV 4000).

Image transfer is not essential for normal (day-to-day) use of Signa Tutor, since a pool of images has been acquired, converted, and stored on the hard drive as an image base. To reference these images by their acquisition parameters, an image database was developed. This data base was written using Oracle (Oracle Corp, Redwood Shores, CA ) version 1.0 and is accessible through the Signa Tutor. The Signa Tutor interface was written in HyperCard version 1.2.2. External functions were written to validate string entry as well as to display the images using the Macintosh Programmer's Workshop ' $\mathrm{C}$ ' development environment. Another program also written in C, called XFORM, was developed to 
convert native Signa images into a Macintosh usable form (Picture format [PICT]) as well as to extract pertinent parameter information from the GE-Signa image header and download it into the image data base.

An important aspect of Signa Tutor is image storage. Since GE-Signa images are 143 Kbytes in size, it is important to minimize the number of images stored. Currently, the images are stored in two forms: PICT version 2, and native Signa format. PICT version 2 is a color version of a Macintosh specific file format and it consists of color lookup table information as well as $256 \times$ $256 \times 8$ bit image data information. The result of PICT conversion is an image file approximately $64 \mathrm{Kbytes}$ in size, a data reduction of $55 \%$. This conversion is performed using the XFORM image conversion program. The XFORM program provides two functions that read the GE image headers and download image parameters into the image database, and convert images to PICT format (when necessary). Some images are saved as native Signa format since one of the external functions computes an image from a set of three images, in native format, to produce a resultant image (described in section 4.2.2, Contrast).

\section{PROGRAM FLOW}

The HyperCard cards within the Signa Tutor stack correspond to GE-Signa scan operation interface screens. At the onset of the stack, there are preliminary cards containing information on how the learner may operate the simulator along with accessibility to preference settings. The preliminary cards perform two purposes: (1) to guide the learner into various modes of scan operation, and (2) to provide a method of selecting a specific anatomical location. The simulator mimics version 3.2 Performance Plus of the GE-Signa scan operation interface. Each screen of the Signa Tutor is a near perfect replica of the Signa console, supporting selection compatibility checking as well as providing messages in a Signa-like fashion. Signa Tutor allows the learner to page through the Signa screens and actually perform an acquisition within a few seconds, as compared with several minutes using a clinical system. The time delay between setting parameters and viewing the image is reduced using Signa Tutor and, therefore, parameter review is not required before proceeding to the next acqui- sition. Thus, the learner has the ability to learn more in a shorter time frame.

As scan parameters are being selected, page checks are made to determine whether images are available for display. These checks occur when the learner moves to the next Signa screen. A select statement is constructed with the current screen's parameters and an image data base check is performed via Oracle. This operation is completely transparent to the learner except when an error, such as no available image, occurs. If such an error occurs, the learner receives an appropriate message indicating a possible reason for and solution to that error.

If the learner successfully traverses SignaTutor, an image corresponding to the parameters that she selected is displayed. These images are displayed using external functions in two forms: directly as a PICT image or computed from three native images and the resultant image displayed (described in section 4.2.2, Contrast).

\section{OPERATION MODES}

Signa Tutor was designed to operate in two modes, an unconstrained mode or a constrained mode. The unconstrained mode permits the learner to make arbitrary choices of scan parameters to form an image. It is comprised of both "Create A Scan" and demonstration modes. Demonstration mode automatically pages, screen by screen, through "Create A Scan" for the learner to view. The constrained mode was developed to teach the learner a specific concept by constraining their parameter selections within Signa Tutor. This mode is comprised of learning modules (Fig 1).

\section{Unconstrained Mode}

With unconstrained mode, "Create A Scan," the learner is shown an image based upon three selection criteria: anatomical location, scan plane, and scan timing parameters. Signa Tutor ignores other selections as nonrelevant information. If the selected imaging parameters are in conflict with each either GE-specific rules or MR imaging fundamentals, ${ }^{1}$ Signa Tutor will give an appropriate error message as defined by the GE-Signa interface. Using "Create A Scan," there are four anatomical location choices available: brain, knee, spine and pelvis. Also three traditional scan planes are available: axial, sagit- 


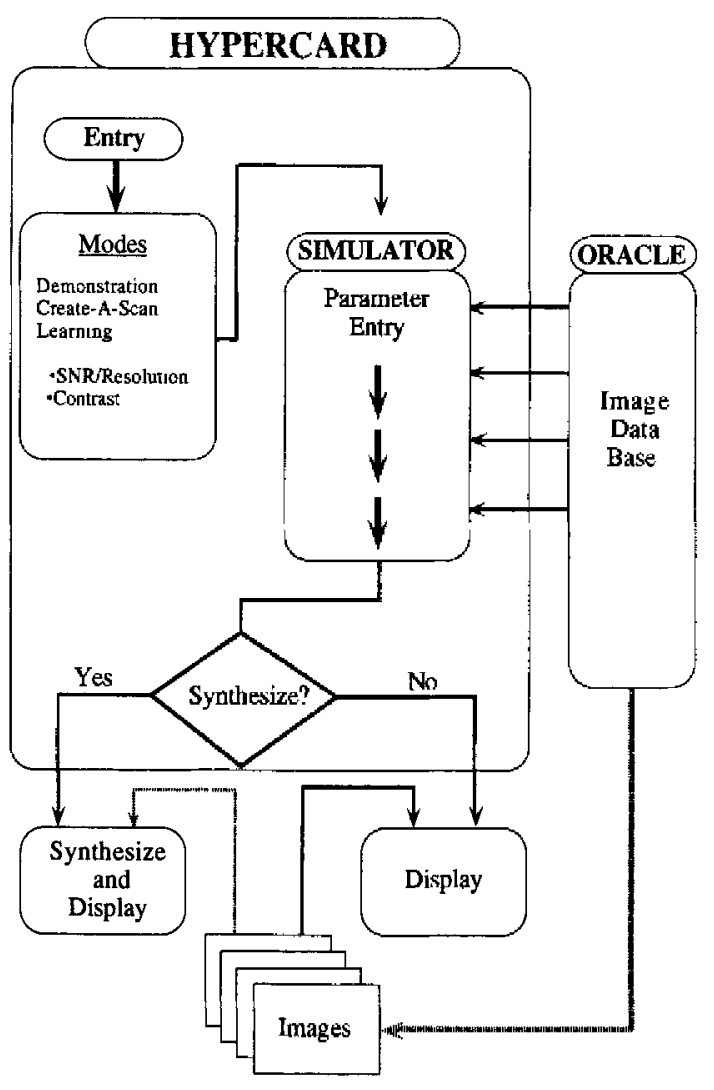

Fig 1. Signa Tutor program flow. First, an operation mode is selected. Then parameters are selected via the simulator and simultaneous database validation is performed. If "Contrast" is selected, images are synthesized and then displayed, otherwise a single PICT image is displayed.

tal and coronal. Finally, three image types are available: $\mathrm{T} 1, \mathrm{~T} 2$, and proton density (PD) weighted images. These image types are specified by a range of repetition (TR) time and echo time (TE) values. A T1-weighted image is displayed when a short TE and a short TR are selected. A T2-weighted image is displayed when a short TE and a long TR are selected. A proton densityweighted image is displayed when a long TE and a long TR are selected (Table 1).

\section{Constrained Mode}

Unlike "Create A Scan," the constrained mode is designed to familiarize the learner with partic-

Table 1. Create A Scan Image Types and Their Associated Timing Parameters

\begin{tabular}{lcl}
\hline Type & TE (msec) & \multicolumn{1}{c}{ TR $(\mathrm{msec})$} \\
\hline T1 & $\leq 30$ & $\geq 30 \& \leq 800$ \\
T2 & $\leq 40$ & $\leq 1500 \& \leq 4000$ \\
PD & $>40$ & $\geq 1500 \& \leq 4000$ \\
\hline
\end{tabular}

ular aspects of MR-imaging in the form of learning modules. At present, two learning modules, "Signal to Noise/Spatial Resolution" and image "Contrast" (pertaining to "Contrast" between tissue due to inherent tissue properties), ${ }^{2,3}$ are available.

\section{Signal to Noise/Spatial Resolution}

The first learning module, "Signal to Noise/ Spatial Resolution", demonstrates the effects of four parameters on image acquisition with regard to image spatial formation and quality. A single scan plane: sagittal, and two anatomical locations: brain and knee are available. The parameters affecting signal to noise and spatial resolution are: field of view, scan thickness (or slice thickness), number of excitations (NEX number of times a slice is measured and averaged), and acquisition matrix (spatial sampling). This image set was produced from a single patient, at the parameters parameters described above, resulting in the acquisition of 108 images (see Fig 2).

\section{Contrast}

The second learning module, "Contrast", illustrates visual differences in gray levels between tissues due to inherent tissue properties. "Contrast" differs from other Signa Tutor modes in that a single image plane can be synthesized following the concept of MR image synthesis. ${ }^{3,4}$ This concept is based on three properties intrinsic to the image, $\mathrm{T} 1, \mathrm{~T} 2$, and proton density, which can be computed from a series of images, taken from a single scan plane of one patient. ${ }^{2}$ These three images are produced on the Signa as a set of three basis images. Once these basis images are available, any image can be synthesized as a resultant image from a function of these three

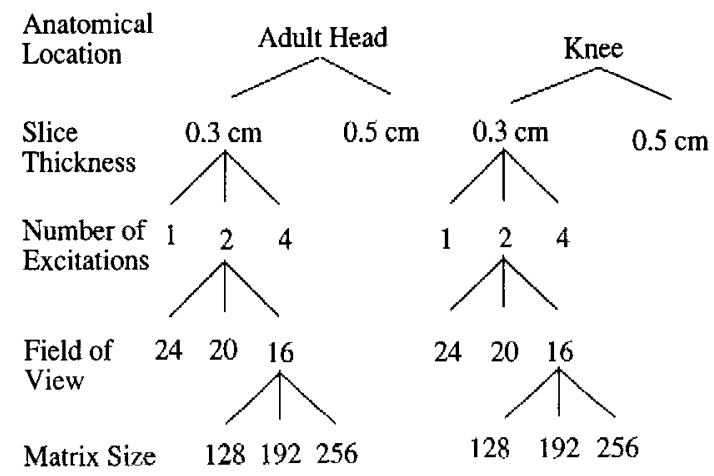

Fig 2. Signal to Noise/Spatial Resolution imege series. 


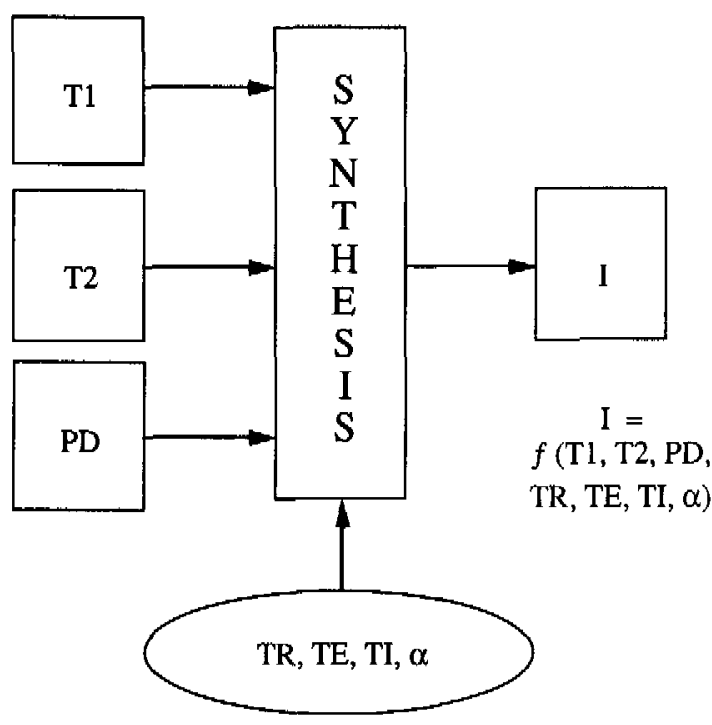

Fig 3. Image synthesis flow diagram. First, the intrinsic properties of a slice plene are computed, (T1, T2, and proton denaity). Then extrinsic pulse sequence timing parameters may selected via the simulator, (TR, TE, TI, and $\alpha)$. An image can be computed as a function of the intrinsic and extrinsic parameters. See text for variable definitions.

intrinsic properties and extrinsic parameters such as pulse sequence timing parameters (Fig 3).

Others have used plots ${ }^{3,5}$ to illustrate the differences in signal intensity of tissues at different timing parameters. These plots make extrapolation to resultant images exceeding difficult to learners since they must integrate two or more plots and are limited to two or three tissues.

With image "Contrast" as presented through Signa Tutor, the learner may see the results of changes in pulse sequences and timing parameters, on one anatomical slice location. The learner may select the following timing parameters: (1) TR and TE are available for all pulse sequences, (2) inversion time (TI) for inversion recovery,
Table 2. Pulse Sequences Used in the "Contrast" Module and Their Associated Timing Parameters

\begin{tabular}{lcccc}
\hline \multicolumn{1}{c}{ Pulse sequence } & \multicolumn{4}{l}{ Parameters } \\
\hline & TR & TE & TI & $\alpha$ \\
SpIn echo & $\mathrm{x}$ & $\mathrm{x}$ & & \\
Inversion recovery & $\mathrm{x}$ & $\mathrm{x}$ & $\mathrm{x}$ & \\
Gradient echo & $\mathrm{x}$ & $\mathrm{x}$ & & $\mathbf{x}$ \\
\hline
\end{tabular}

Abbreviation: $\alpha$, flip angle.

and (3) flip angle ( $\alpha$ ) for a gradient echo pulse sequence (Table 2 ).

Spatial orientations available for "Contrast" mode are three anatomical locations: brain, knee, and pelvis, and three scan planes: axial, sagittal, and coronal. The other parameters affected are the pulse sequence and the timing parameters. Therefore, if a spin echo pulse sequence is chosen, TR and TE affect the image. If an inversion recovery pulse sequence is selected, a new parameter TI affects the image along with TR and TE. Similarly, if a gradient echo pulse sequence is selected, $\alpha$ will affect the image along with TR and TE.

\section{CONCLUSION}

An MR imaging simulator, Signa Tutor, has been developed. It has been used in conjunction with MRI training courses, and has received a large degree of enthusiasm and acceptance from the students. At present, quantitative measures of acceptance or proof of effectiveness in learning have not yet been established. Future versions of Signa Tutor will support releases of GE-Signa software, including recently released version 4.0.

\section{ACKNOWLEDGMENT}

We would like to thank Carolyn Watson, Larry Dougherty, and Dr Philip Chao for their contributions to the design and development of Signa Tutor, and all of the MRI technicians for their assistance in testing Signa Tutor.

\section{REFERENCES}

1. Dixon RL, Ekstrand KE: Physical Foundations of Proton NMR, Part 1: Thomas SR and Dixon RL: NMR in medicine: The Instrumentation and Clinical Applications. American Institute of Physics, New York, NY, 1986 pp 1-31

2. Wehrli FW, MacFall JR, Glover GH, et al: The dependence of nuclear magnetic resonance (NMR) image contrast on intrinsic and pulse sequence timing parameters. Magn Reson Imaging 2:3-16, 1984
3. Wehrli FW, Shaw D, Kneeland JB: Biomedical Magnetic Resonance Imaging. VCH, New York, NY, 1988

4. Riederer SJ, Suddarth SA, Bobman SA, et al: Automated MR image synthesis: Feasibility studies. Radiology 153:203-206, 1984

5. Posteraro R, Blinder R, Herfkens R: MR-Tutor: A program for teaching the interdependence of factors which influence signal intensity in magnetic resonance imaging. Computerized Med Imag and Graph 13:393-406, 1989 